\title{
OPEN Validation of galectin-1 as potential diagnostic biomarker of early rheumatoid arthritis
}

\begin{abstract}
Ana Triguero-Martínez ${ }^{1}$, Hortensia de la Fuente ${ }^{2}$, Nuria Montes ${ }^{1}$, Ana María Ortiz ${ }^{1}$, Emilia Roy-Vallejo ${ }^{3}$, Santos Castañeda ${ }^{1}$, Isidoro González-Alvaro ${ }^{1 \bowtie}$ \& Amalia Lamana ${ }^{4 \bowtie}$

Galectin 1 (Gal1) is a lectin with a wide cellular expression that functions as a negative regulator of the immune system in several animal models of autoimmune diseases. Identification of patients with rheumatoid arthritis (RA) has improved during the last decade, although there is still a need for biomarkers allowing an early diagnosis. In this regard, it has been recently proposed that Gal1 serum levels are increased in patients with RA compared to the general population. However, this topic is controversial in the literature. In this work, we provide additional information about the potential usefulness of Gal1 serum levels as a biomarker for RA diagnosis. We studied Gal1 serum and synovial fluid levels and clinical parameters in samples from 62 patients with early arthritis belonging to the PEARL study. In addition, 24 healthy donors were studied. We found that both patients fulfilling RA criteria and patients with undifferentiated arthritis showed higher Gal1 levels than healthy donors. Similar findings were observed in synovial fluid, which showed even higher levels than serum. However, we did not find correlation between Gal1 levels and disease activity or disability. Therefore, our results suggest that Gal1 could be a diagnostic but not a severity biomarker.
\end{abstract}

Rheumatoid arthritis (RA) is the most common autoimmune rheumatic disease. Although the ethiopathogenesis of RA has not been fully elucidated, it is considered a complex disease in which the interaction between genetic, environmental and stochastic factors leads to a chronic infiltration of the synovial membrane by a variety of cells of the immune system. These infiltrated cells cause chronic production of proinflammatory cytokines (TNF- $\alpha$, IL-1 $\beta$ and IL-6) and metalloproteinases that usually leads to joint destruction due to cartilage degradation and bone erosion ${ }^{1}$.

During the last years, solid evidence has confirmed the "window of opportunity" concept, which establishes the possibility of reaching better outcomes with early treatment with disease modifying anti-rheumatic drugs (DMARDs) $)^{2,3}$. On the other hand, a better knowledge of the regulation of the immune system can improve our ability to tailor treatment intensity. At present, it is accepted for seropositive RA that the interaction between HLA DRB1 alleles encoding the "shared epitope" and smoking habit increases the risk of developing autoreactive $\mathrm{T}$ and $\mathrm{B}$ clones. These autoreactive clones in turn produce antibodies against citrullinated proteins (ACPA), which lead to chronic synovitis ${ }^{4}$. However, more than 100 loci have been suggested to contribute to the genetic risk of RA development ${ }^{5}$ and several environmental factors ${ }^{4}$ may also interact with them to promote RA, likely leading to a very heterogeneous clinical course.

Although some experts have suggested to treat intensively arthritis to abrogate inflammation ${ }^{6}$, it seems wise to avoid over-treating patients less likely to develop a severe disease ${ }^{7}$. In this regard, the landscape can be even more complex, since we have recently described that genetic variants in the region encoding for vasoactive intestinal peptide (VIP), which have not been associated with the risk of developing RA, contribute to the heterogeneity of disease severity ${ }^{8}$. Therefore, the ability to predict the heterogeneous clinical spectrum of RA has improved with the introduction of $\mathrm{ACPA}^{9}$, although it is still low due to our limitations in understanding its molecular complexity.

Galectins are lectins that bind $\beta$-galactoside carbohydrates ${ }^{10,11}$ via carbohydrate recognition domains (CRDs). At least 15 mammalian galectins have been described, all containing 1 or 2 CRDs of approximately 130 amino

\footnotetext{
${ }^{1}$ Rheumatology Department, Hospital Universitario La Princesa, Instituto de Investigación Sanitaria La Princesa (IIS-IP), Madrid, Spain. '2Immunology Department, Hospital Universitario La Princesa, Instituto de Investigación Sanitaria La Princesa (IIS-IP), Madrid, Spain. ${ }^{3}$ nnternal Medicine Service, Hospital Universitario La Princesa, Instituto de Investigación Sanitaria La Princesa (IIS-IP), Madrid, Spain. ${ }^{4}$ Cell Biology Department, Facultad de Biología, Universidad Complutense de Madrid, Madrid, Spain. ${ }^{\varpi}$ email: isidoro.ga@ser.es; amaliala@ucm.es
} 


\begin{tabular}{|c|c|c|c|c|}
\hline & Population 1 & Population 2 & Healthy donors & \multirow[b]{2}{*}{$\mathbf{p}$} \\
\hline & $(\mathbf{n}=\mathbf{3 0})$ & $(\mathbf{n}=32)$ & $(\mathbf{n}=\mathbf{2 4})$ & \\
\hline Female; n (\%) & $25(83.33)$ & $27(84.38)$ & $20(83.33)$ & 1 \\
\hline Age; p50 [p25-p75] & $52[39-70]$ & $52[43-60]$ & $52[36-64]$ & 0.79 \\
\hline BMI; p50 [p25-p75] & $26.46[23.5-29.27]$ & $26.78[22.87-30.26]$ & $28.23[25.05-29.37]$ & 0.646 \\
\hline RA/UA; n (\%) & $30(100) / 0(0)$ & $21(65.63) / 11(34.38)$ & & $<0.001$ \\
\hline Disease duration (months); p50 [p25-p75] & $6.48[2.76-8.83]$ & $2.78[2-7.81]$ & & 0.060 \\
\hline RF positive; $n(\%)$ & $22(73.33)$ & $16(50)$ & & 0.059 \\
\hline ACPA positive; $\mathrm{n}(\%)$ & $20(66.67)$ & $16(50)$ & & 0.184 \\
\hline DAS28; p50 [p25-p75] & $5.12[3.89-5.8]$ & $3.91[2.98-6.34]$ & & 0.049 \\
\hline HAQ; p50 [p25-p75] & $1.06[0.62-1.62]$ & $1[0.81-1.37]$ & & 0.970 \\
\hline
\end{tabular}

Table 1. Baseline characteristics of the populations studied. p50: median; p25-p75: interquartile range; BMI: body mass index; RA: rheumatoid arthritis; UA: undifferentiated arthritis; RF: rheumatoid factor; ACPA: anti-citrullinated protein antibodies; DAS28: disease activity score estimated with 28 joint count. HAQ: health assessment questionnaire.

acids. The variety of binding partners and the wide distribution of galectins allow them to participate in multiple biological functions, including the regulation of the immune system ${ }^{12}$.

Galectin 1 (Gal1) is a protein of approximately $14 \mathrm{KDa}$ that is able to form homodimers. It is highly expressed in immune cells and its expression is modulated during activation and differentiation ${ }^{12}$. Gall plays an immunosuppressive and anti-inflammatory role due to its pro-apoptotic effect in activated lymphocytes ${ }^{13}$, promoting the polarization of Th1 responses towards Th2 ${ }^{14}$ and Th17 towards Treg ${ }^{15}$, as well as blocking the in vitro secretion of pro-inflammatory cytokines (IL-2, IFN $\gamma$ and TNFa) ${ }^{16,17}$. In addition, treatment with recombinant Gall improves clinical manifestations of established collagen-induced arthritis (CIA $)^{15}$. A recent cross-sectional study described that RA patients show higher Gall serum levels ${ }^{18}$. However, other authors have described similar Gall serum levels in RA patients and healthy controls ${ }^{19}$.

Additional controversial data have been reported describing reduced expression of Gall in synovial tissue of patients with juvenile idiopathic arthritis ${ }^{20}$, absence of Gall expression at cartilage invasion sites in RA patients ${ }^{21}$ or decreased Gall levels in synovial fluid in comparison to healthy controls ${ }^{19}$.

Therefore, the role of Gall as a biomarker for RA remains controversial. In this work, we aim to describe the evolution of Gall serum level in a longitudinal study in patients with early arthritis (EA), as well as to compare Gal1 levels in synovial fluid from patients with different inflammatory arthritis and osteoarthritis.

\section{Results}

Study population. In order to validate the report by Mendez-Huergo et al. describing that patients with RA show higher Gall serum levels than healthy donors ${ }^{18}$, we measured Gall levels in serum from 62 patients included in the PEARL study. Patients were clustered in two different populations (Pop1 and Pop2) and 24 healthy donors (HD) were also studied. The characteristics of the 3 populations are shown in Table 1 . No significant differences were found between populations. The only difference was that Pop1 included 30 RA patients, while Pop2 was a mixed population with a $34 \%$ of undifferentiated arthritis (UA); therefore, a significantly higher baseline DAS28 was observed in Pop1.

Early arthritis patients show higher Gal1 serum levels than healthy controls. As it is shown in Fig. 1A, baseline Gal1 serum levels in RA patients of Pop1 were significantly higher than levels of healthy controls. Furthermore, this increased expression was maintained all along the follow-up and samples from all 4 visits of patients showed significantly higher Gall levels than samples from healthy controls (baseline $\mathrm{p}=0.007$; 6 months $\mathrm{p}<0.001 ; 12$ months $\mathrm{p}=0.040$; and 24 months $\mathrm{p}=0.008$; Fig. 1A). Furthermore, a Gal1 serum concentration above $19.12 \mathrm{ng} / \mathrm{ml}$ could differentiate RA patients from controls (AUC $=0.761$ ) with $71 \%$ sensitivity and 79\% specificity (Fig. 1C; LR+3.44 and LR- 0.35).

In addition, we were interested in whether the evolution of Gall levels was different in RA patients versus those with UA. Our data showed no significant differences between the baseline expression levels of RA patients with respect to UA, with a poor capability to differentiate between RA and UA (AUC $=0.55$ ), being the best cut-off point $34 \mathrm{ng} / \mathrm{ml}$ with a $27 \%$ sensitivity and $96 \%$ specificity (LR+6.81, LR- 0.76 ; Fig. 1D). Although no significant down regulation of Gall was observed throughout the follow-up either in RA or in UA, a slightly higher Gal1 was observed in serum from patients with RA at the 2 -year visit $(p=0.08$, Fig. 1B).

Gal1 serum levels are not associated with activity or disability in EA patients. To determine whether Gal1 serum levels could be a biomarker of severity, we studied their correlation with either disease activity assessed by DAS28 or disability measured by HAQ score. As it is shown in Fig. 2A,B, disease activity decreased along the follow-up in both Pop1 (Fig. 2A) and Pop2 (Fig. 2B) in response to treatment (Table S2). However, Gall levels remained elevated without significant fluctuations throughout the follow-up (Fig. 1A,B). Therefore, no significant correlation was observed between DAS28 and Gal1 serum levels in both populations 
A

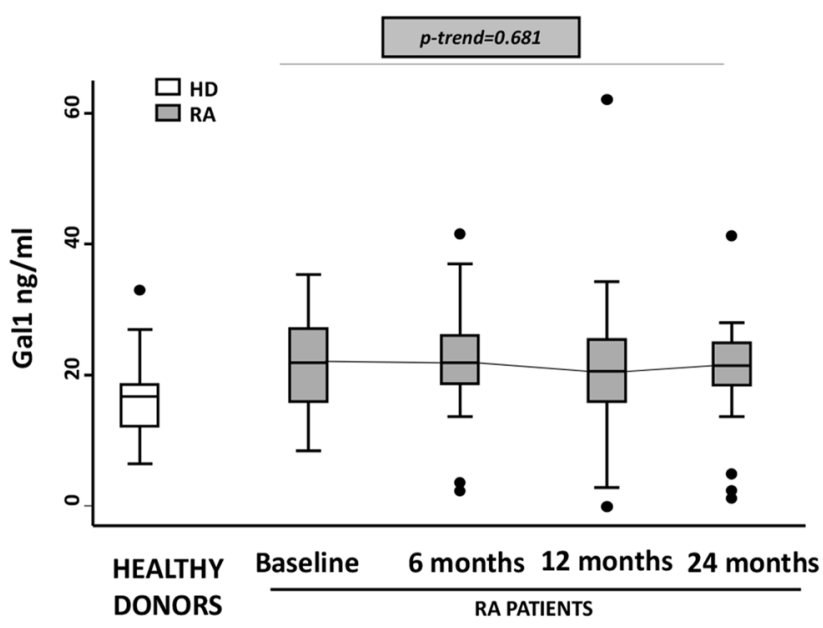

\begin{tabular}{|c|c|c|c|c|}
\hline & VISIT & median & IQR & $\begin{array}{l}\text { RA vs healthy } \\
\text { donors }\end{array}$ \\
\hline $\begin{array}{l}\text { HEALTHY } \\
\text { DONORS }\end{array}$ & & 16.67 & $12.12 \quad 18.52$ & $P$ \\
\hline \multirow{4}{*}{ RA PATIENTS } & Baseline & 21.84 & $\begin{array}{ll}15.87 & 27.09\end{array}$ & 0.007 \\
\hline & 6 months & 21.83 & $18.62 \quad 26.04$ & $<0.001$ \\
\hline & 12 months & 20.54 & $15.88 \quad 25.38$ & 0.040 \\
\hline & 24 months & 21.41 & $18.40 \quad 24.93$ & 0.008 \\
\hline
\end{tabular}

C

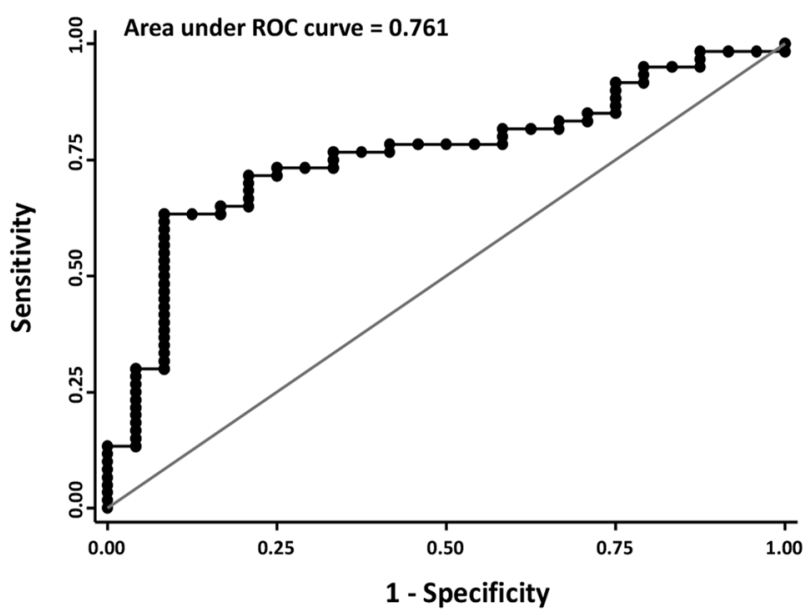

B

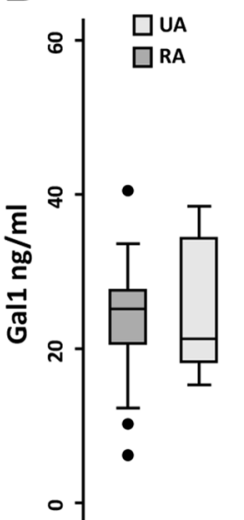

Population 2

$p$-trend $=0.470$

p-trend $=0.140$

$\boxminus \boxminus$

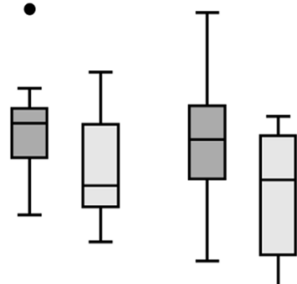

Baseline

6 months

12 months

24 months

\begin{tabular}{|c|ccccc|}
\cline { 2 - 6 } \multicolumn{1}{c|}{} & DX & median & IQR & $\begin{array}{c}\boldsymbol{p} \\
\text { RA Vs UA }\end{array}$ \\
\hline & RA & 25.18 & 20.64 & 27.56 & \\
Baseline & UA & 21.23 & 18.26 & 34.29 & 0.939 \\
\hline & RA & 24.58 & 21.43 & 27.53 & \\
6 months & UA & 20.77 & 18.52 & 26.57 & 0.289 \\
\hline & RA & 26.66 & 22.21 & 28.54 & \\
12 months & UA & 18.55 & 18.85 & 28.74 & 0.186 \\
\hline & RA & 24.54 & 19.47 & 28.94 & \\
24 months & UA & 19.34 & 9.52 & 24.99 & 0.082 \\
\hline
\end{tabular}

D

RA vs UA patients

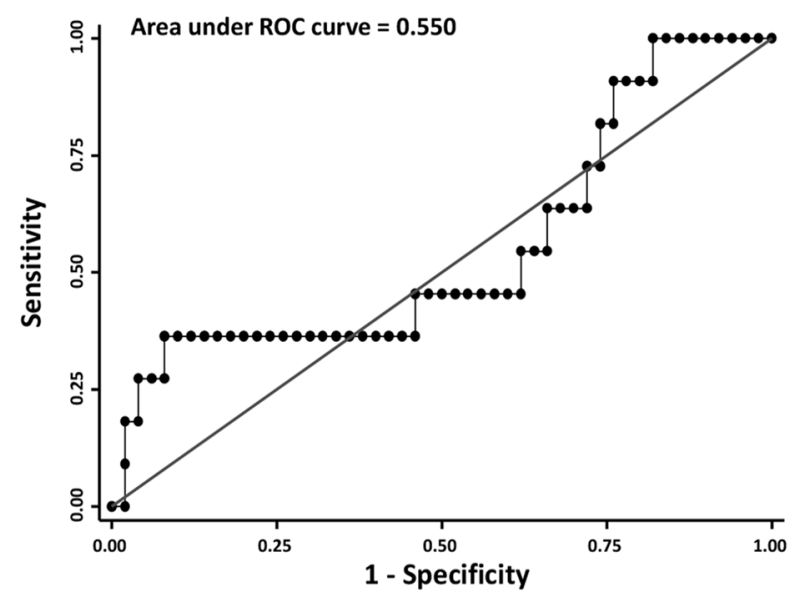

Figure 1. Gall serum levels are increased in early arthritis patients compared with healthy donors. (A) Determination of Gal1 serum levels by ELISA in healthy donors (HD) and rheumatoid arthritis (RA) patients from PEARL study (Population 1). (B) Determination of Gall serum levels by ELISA in early arthritis patients (undifferentiated arthritis [UA] and RA) from PEARL study (Population 2). Data are shown as interquartile range (p75 upper edge of box, p25 lower edge, p50 midline) as well as the p95 (line above box) and p5 (line below). Dots represent outliers. Statistical significance for the trend of Gall across the visits in patients was determined with the Cuzick's non-parametric test. Significance threshold was set at $\mathrm{p}$-trend $<0.05$. Tables below the panels show the statistical significance between HD and each visit of RA patients (A) or between RA and UA patients in each visit (B). Statistical significance was determined with Mann-Whitney test. Significance threshold was set at $\mathrm{p}<0.012$ due to multiple comparisons, according to Bonferroni correction. (C) ROC curve analysis to assess Gall capacity to discriminate between RA patients and healthy donors. (D) ROC curve analysis to assess Gall capacity to discriminate between RA patients and UA patients. 

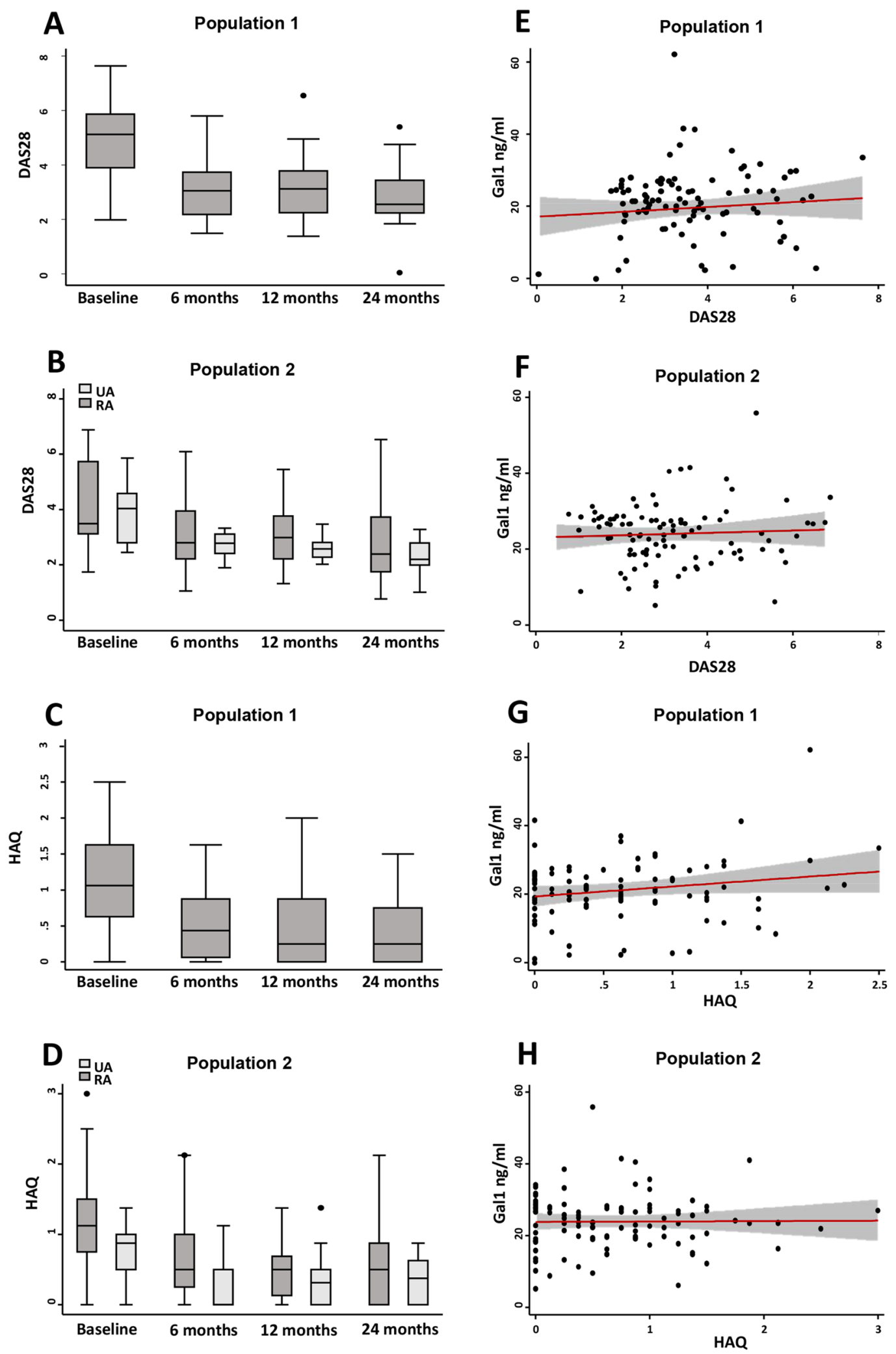

Figure 2. Gall serum levels do not correlate with clinical severity parameters in early arthritis. Evolution along the follow-up of disease activity assessed by DAS28 (A Population 1; B Population 2) and disability assessed by HAQ (C Population 1; D Population 2) in early arthritis. Absence of correlation of Gall serum levels with DAS28 (E Population 1; F Population 2) and HAQ (G Population 1; H Population 2) in early arthritis. In (A-D), data are shown as interquartile range (p75 upper edge, p25 lower edge, p50 midline), p95 (line above the box) and p5 (line below the box). Dots represent outliers. In (E-H), data are shown as dot-plots and their fitted linear prediction with 95\% confidence interval (grey shadow) estimated using the twoway command of Stata with the litci option. Pearson correlation test was used to determine the level of significance. 


\begin{tabular}{|c|c|c|}
\hline & \multicolumn{2}{|l|}{ Disease activity } \\
\hline & $\beta$ Coeff. $(95 \% \mathrm{CI})$ & p value \\
\hline DAS28 & $0.51(-0.32$ to 1.36$)$ & 0.231 \\
\hline Female gender & $2.15(-1.5$ to 5.8$)$ & 0.256 \\
\hline \multicolumn{3}{|l|}{ Age (years) } \\
\hline$<45$ & Reference & - \\
\hline $45-65$ & 4.68 (1.40 to 7.95$)$ & 0.005 \\
\hline$>65$ & 2.87 ( -0.64 to 6.39$)$ & 0.109 \\
\hline \multirow[t]{3}{*}{ Time of frozen storage (months) } & $-0.03(-0.06$ to -0.01$)$ & 0.002 \\
\hline & \multicolumn{2}{|l|}{ Disability } \\
\hline & $\beta$ Coeff. $(95 \%$ CI $)$ & p value \\
\hline HAQ & $1.26(-0.62$ to 3.15$)$ & 0.189 \\
\hline Female gender & $1.87(-1.93$ to 5.67$)$ & 0.336 \\
\hline \multicolumn{3}{|l|}{ Age (years) } \\
\hline$<45$ & Reference & - \\
\hline $45-65$ & 4.55 (1.29 to 7.81$)$ & 0.006 \\
\hline$>65$ & $2.54(-1.06$ to 6.14$)$ & 0.167 \\
\hline Time of frozen storage (months) & $-0.03(-0.06$ to -0.01$)$ & 0.005 \\
\hline
\end{tabular}

Table 2. Relationship between Gall (ng/ml) serum levels and disease activity or disability. DAS28, 28-Joint Disease Activity Score; HAQ, Health Assesment Questionnaire; Coeff. Coefficient.
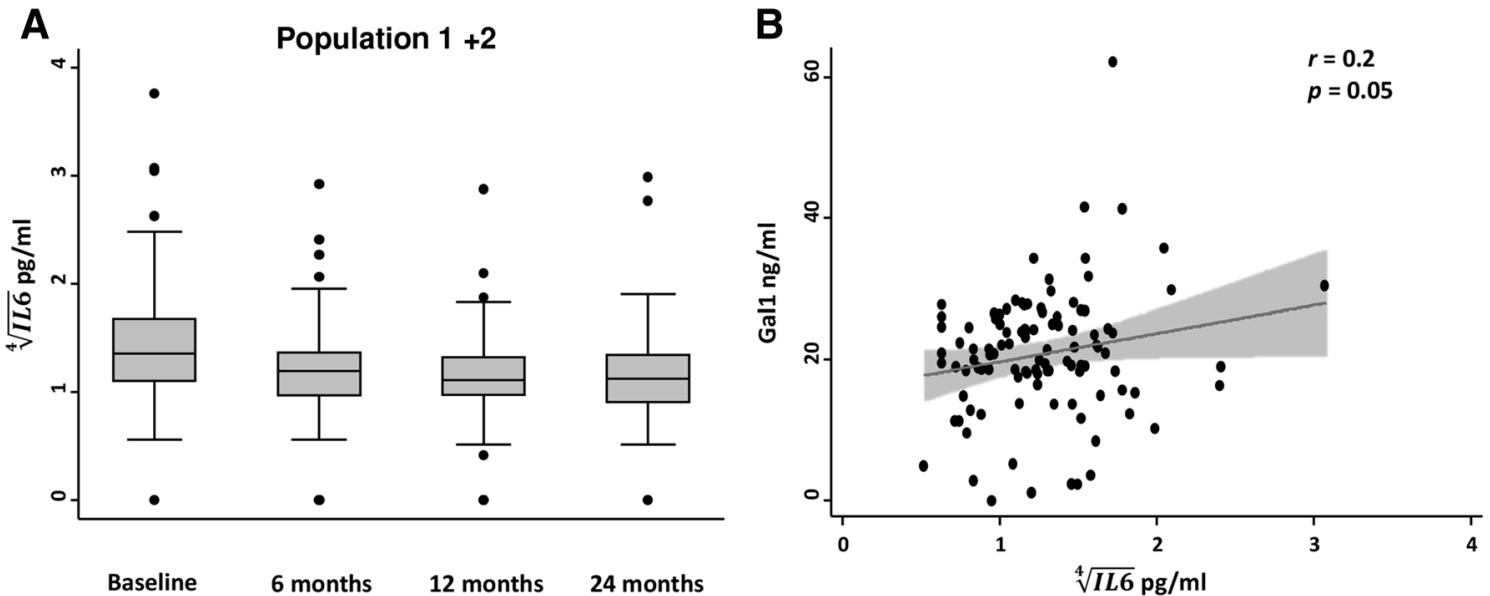

Figure 3. Gall serum levels correlate with IL-6 serum levels in early arthritis. (A) Evolution along the follow-up of IL6 serum levels (Population 1 plus Population 2) in early arthritis patients. Data are shown as interquartile range (p75 upper edge, p 25 lower edge, p50 midline), p95 (line above the box) and p5 (line below the box).Dots represent outliers. (B) Moderate correlation between Gal1 and IL6 serum levels (Population 1 plus Population 2) in early arthritis patients. Data are shown as dot-plots and their fitted linear prediction with $95 \%$ confidence interval (grey shadow) estimated using the twoway command of Stata with the lfitci option. Pearson correlation test was used to determine the level of significance.

(Fig. 2E,F). A similar finding was observed with disability, HAQ score improved throughout the follow-up (Fig. 2C,D), whereas no significant correlation was observed between HAQ and Gall serum levels (Fig. 2G,H).

Also, we performed a multivariate analysis to determine which variables could be associated with Gall serum levels. We observed that age and female gender positively correlated with Gall levels (Table 2), while, as mentioned above, time of frozen storage negatively correlated in a very significant way (Table 2 and Fig. S1). After adjustment for these confounding variables, the analysis confirmed that neither DAS28 nor HAQ (Table 2, upper and lower sections of the table, respectively) correlated with Gall serum levels throughout the follow-up.

Gal1 serum levels correlate with serum IL6 in RA. Since it has been described that Gal1 is able to decrease IL6 production in vitro ${ }^{22}$, we were interested in determining whether Gall was associated with the serum levels of the proinflammatory cytokine IL6 in our EA patients. As observed above for disease activity and disability, IL6 levels decreased along the follow-up (Fig. 3A). Nevertheless, we observed a moderate, but significant correlation between Gal1 serum levels and IL6 levels ( $r=0.2, p=0.05$; Fig. 3B). To confirm that this 


\begin{tabular}{|c|c|c|}
\hline & $\beta$ Coeff. $(95 \%$ CI $)$ & p value \\
\hline Galectinl (ng/ml) & $0.008(0.001$ to 0.01$)$ & 0.023 \\
\hline \multicolumn{3}{|l|}{ Age (years) } \\
\hline$<45$ & Reference & - \\
\hline $45-65$ & $0.18(-0.06$ to 0.43$)$ & 0.154 \\
\hline$>65$ & $0.17(-0.06$ to 0.40$)$ & 0.162 \\
\hline \multicolumn{3}{|l|}{ DAS28 } \\
\hline Remission & Reference & - \\
\hline Low DA & $0.09(-0.09$ to 0.27$)$ & 0.327 \\
\hline Moderate DA & $0.31(0.15$ to 0.47$)$ & $<0.001$ \\
\hline High DA & $0.41(0.18$ to 0.64$)$ & $<0.001$ \\
\hline Methotrexate yes/no & $-0.16(-0.31$ to -0.004$)$ & 0.044 \\
\hline
\end{tabular}

Table 3. Relationship between IL6 (pg/ml) and Gall (ng/ml) serum levels. DAS28: disease activity score estimated with 28 joint count; Coeff., coefficient; IL6, interlukin 6; DA: disease activity.

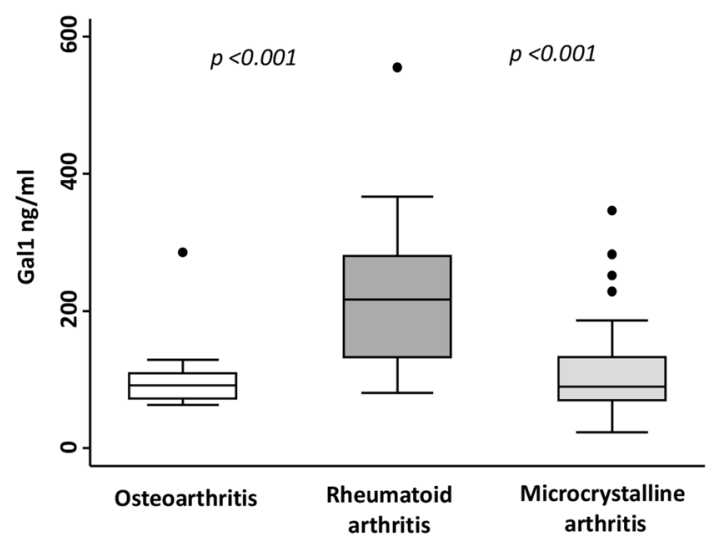

Figure 4. Gall synovial fluid levels are increased in RA patients. Determination of Gall synovial fluid levels by ELISA in OA, microcrystalline arthritis and RA patients. Data are shown as interquartile range (p75 upper edge of box, p25 lower edge, p50 midline) as well as the p95 (line above box) and p5 (line below). Dots represent outliers. Statistical significance was determined with ANOVA. Significance threshold was set at $\mathrm{p}<0.05$.

association was not due to some bias, we performed a multivariate analysis in which, as expected, we observed that IL6 serum levels positively correlated with levels of disease activity and with increasing age. Conversely, methotrexate treatment was associated with decreased IL6 levels (Table 3), as previously described ${ }^{23,24}$. After adjustment for these variables a significant association between Gall and IL6 serum levels was still observed (Table 3) confirming the correlation showed in Fig. 3B.

Gal1 synovial fluid levels are increased in RA patients. Finally, we were interested in determining whether Gall was also locally increased in patients with RA. Thus, we studied Gal1 levels in SF from 20 patients with RA, 20 with osteoarthritis and 22 with microcrystalline arthritis (gouty and chondrocalcinosis arthritis).

We observed that RA patients had significantly higher Gall levels than OA patients $(\mathrm{p}<0.001$, Fig. 4) and patients with microcrystalline arthritis $(\mathrm{p}<0.001$, Fig. 4). Furthermore, in six RA patients, not included either in pop1 or in pop2, we had the opportunity to obtain in parallel SF and blood samples and the levels of Gall in SF were significantly higher than those detected in serum, $(\mathrm{p}<0.001$, Fig. S2).

\section{Discussion}

The main findings of this work are: (i) the validation of serum Gall measurement as a possible diagnostic biomarker for RA; and (ii) the presence of even higher levels of this molecule in synovial fluid from patients with RA, as well as in comparison with synovial fluid from other mechanic or inflammatory processes such as osteoarthritis and microcrystalline arthritis.

The current paradigm of RA management is to achieve remission as soon as possible ${ }^{25}$. However, despite the new classification criteria for RA including rheumatoid factor and anti citrullinated protein antibodies as diagnostic biomarkers ${ }^{26}$, many patients at early arthritis clinics do not fulfil these criteria. Many efforts have been made to improve the accuracy of these classification criteria, especially including new autoantibodies, such as anti carbamylated protein antibodies ${ }^{27}$.

In this regard, until now, the available data on Gall serum levels in RA patients and their possible correlation with clinical severity parameters were controversial. The first study comparing Gall serum levels from RA 
patients and HD found no differences between them ${ }^{19}$. On the other hand, the recent article by Mendez-Huergo et al. described that Gall serum levels were higher in RA patients than in HD and correlated with clinical severity parameters ${ }^{18}$. Our study supports the later and, additionally, it has the advantage that it was performed in patients with early arthritis using a longitudinal design. This design allowed to analyse Gall levels at the beginning of the disease in untreated patients (visit 1), as well as during their follow-up after the establishment of the treatment. A baseline Gall serum level $>19 \mathrm{ng} / \mathrm{ml}$ showed a good performance to discriminate HD from either RA or UA patients. The difference between the cut-off point proposed in the study by Mendez-Huergo et al. and our study could be due to the use of different ELISA kits to measure Gal1 in serum ${ }^{18}$. In our case, we used a commercial kit, which showed very low variability between tests. The use of standardized ELISA kits instead of home-made kits would be a good strategy since a fundamental point in the search for biomarkers and their subsequent validations is reproducibility.

The other point of controversy is whether Gal-1 levels may be a severity biomarker or could be considered as an acute phase reactant. The only work that tried to address this point was that of Mendez-Huergo et al., which only found a mild correlation with parameters of disease activity ${ }^{18}$. Our work did not show association with disease activity or disability. Despite these outcomes improved with treatment along the follow-up, no significant decrease in Gal-1 serum levels was observed. Furthermore, no association with severity outcomes, such as achievement of remission, radiological progression, cumulative intensity of treatment or need for biological therapy was detected. Additional studies with sera from different inflammatory arthropathies would be necessary to determine whether increased serum levels of Gal-1 are RA specific and can be used as a diagnostic biomarker.

To gain further insight into the potential diagnostic utility of Gall, we measured its expression in SF from different pathologies. Previously, Xibille-Friedmann et al. observed that the level of Gall was significantly lower in RA patients than in patients with non-arthritic knee effusion ${ }^{19}$. On the contrary, our data show that Gal1 synovial levels in RA are higher compared to osteoarthritis and microcrystalline arthritis. These different results could be explained by the treatment of SF samples with hyaluronidase in the work of Xibille-Friedmann et al. ${ }^{19}$, which could have interfered with their measurement of Gall in SF. In addition, they did not take into account the time of frozen storage of the samples, which is a variable that can significantly affect Gall detection, as we have demonstrated here. Finally, in their study, low levels of Gall correlated with the presence of anti-Gall antibodies in the joint ${ }^{19}$, suggesting that a technical interference between the antibodies of their ELISA kit and the possible presence of anti-Gall antibodies may have occurred in their experiments. In addition, our study supports a local production of Gall in RA patients, since its levels were significantly higher in SF than in serum.

Regarding pathogenesis of the disease, IL6 plays an important role in RA, which has allowed the development of IL- 6 signalling blockade as an effective tool in the treatment of RA patients ${ }^{1}$. Treatment with Gall reduces proinflammatory cytokine levels in vitro ${ }^{16,17}$; however, its effect on IL6 production has not been described. Although IL6 serum levels decreased along the follow-up while Gall levels mostly remained unaffected, we were still able to detect a moderate association between them. Therefore, it is likely that Gal1 and IL6 are related in the etiopathogenesis of the RA, at least in a subpopulation of patients. In this regard, the slight differences observed in Gall serum levels between patients with RA and UA at the last visit of follow-up could be due to the heterogeneity of the UA population. Thus, patients not fulfilling RA criteria because they are efficiently treated at very early stages of the disease could behave as RA patients and still display high Gall at this last visit, whilst patients with other disorders may undergo Gall down-regulation once disease activity is controlled. Another explanation could be the low number of UA patients included in the study would not allow the Cuzick's test to detect a significant trend in Gall levels along the follow-up. However, we believe that it would be interesting to continue studying differences in Gall serum levels between RA and UA patients, since Gall levels could be useful for early diagnosis in EA patients. It would also be interesting to unravel the combined role of Gall and IL6 in the pathophisiolgy of RA.

In conclusion, our work suggests that Gall serum levels may have role as a diagnostic biomarker in patients with early RA, although they cannot be considered a severity biomarker. In addition, they support that Gall and IL6 may be related during RA etiopathogenesis. Therefore, our study opens a door to further characterise the role of Gall in the pathogenic basis of RA, especially by exploring its relationship with IL6.

\section{Materials and methods}

Serum sample from early arthritis population. The present study was performed with data and samples from the Princesa Early Arthritis Register Longitudinal (PEARL) study carried out at the Hospital Universitario de La Princesa, Madrid, Spain. The PEARL study comprises patients with one or more swollen joint and symptoms with $\leq 1$ year of evolution. The register protocol includes 4 visits during a 2-year follow-up $(0,6,12$ and 24 months). Socio-demographic, clinical, therapeutic and laboratory data are recorded and included in an electronic database. Biological samples are collected at each visit and stored at $-80^{\circ} \mathrm{C}$ in the Instituto de Investigación Sanitaria La Princesa (IIS-IP) Biobank for translational research. More detailed description of PEARL protocol has been previously published ${ }^{28}$.

At the end of follow-up,all EA patients are classified as RA if they fulfil 1987 ACR classification criteria ${ }^{29}$ or as UA as described by Verpoort et al. ${ }^{30}$. Patients with other defined diagnosis (connective tissue diseases, psoriatic arthritis, gout or osteoarthritis) are excluded from the study.

For this study, two independent PEARL populations of patients were studied. Population 1 (Pop1) comprised 30 RA patients with data from 95 visits (average 3.2 per patient) and Population 2 (Pop2) (validation group) comprising $21 \mathrm{RA}$ and $11 \mathrm{UA}$ patients with data from 107 visits (average 3.2 per patient). Twenty-four healthy donors were also analysed. Clinical characteristics of all populations are shown in Table 1. 
Synovial fluid sample population. Synovial fluid (SF) samples were obtained from therapeutic or diagnostic arthrocentesis after oral informed consent registered in the clinical chart. Those samples contaminated with blood were discarded. Samples were centrifuged at $2000 \mathrm{rpm}$ during $20 \mathrm{~min}$ at room temperature and the cell-free supernatants were collected and stored at $-80^{\circ} \mathrm{C}$ until analysis. The samples were anonymised and only information about diagnosis was collected. For this study, samples from 20 RA patients, 20 osteoarthritis (OA) patients and 22 microcrystalline arthritis patients (gouty and chondrocalcinosis arthritis) were analysed.

Measure of serum and synovial fluid Gal1. Gall serum and SF levels were measured using Quantikine Human Gall Immunoassay (R\&D Systems, Minneapolis, USA). The procedure was performed according to the manufacturer's instructions. Absorbance was measured in a spectrophotometer (Innogenetics Diagnostica y terapeutica S.A.U, Barcelona, Spain) at $450 \mathrm{~nm}$ with correction at $620 \mathrm{~nm}$. Measurements for all samples were performed in triplicate.

Time of frozen storage of the samples. Since time of frozen storage of the samples analysed ranged from 6 to 200 months, we tested whether this circumstance could affect Gall detection. As Supplementary Fig. S1 shows, time of frozen storage significantly affected the capability to detect Gall $\left(\mathrm{p}=1.4710^{-5}\right.$ and $\mathrm{R}^{2}=0.07$ ), although differences between healthy donors and patients were clearly observed even in the samples with longer frozen storage. Therefore, the variable time of frozen storage was used as a confounding variable in the following multivariate analyses.

Statistical analysis. Statistical analyses were performed using Stata 14 for Windows (Stata Corp LP, College Station, TX, USA). Most quantitative variables followed a non-normal distribution, so they were represented as median and interquartile range (IQR) and the Mann Whitney or Kruskal-Wallis tests were used to analyse significant differences. When a variable was normalised (see below) the Student t-test was applied. Qualitative variables were described using a calculation of the proportions and the $\chi^{2}$ or Fisher's exact test was used to compare categorical variables. The Pearson correlation test was applied to analyse correlation between quantitative variables.

The Cuzick's test, an extension of the Wilcoxon rank-sum test, was used to determine statistical significance of the distribution trend across ordered groups in variables such as visits or level of disease activity.

The relationship between time of frozen storage (days) and Gall serum levels was analysed by polynomial second degree regression conducted with $\mathrm{R}$ version $3.5 .0^{31}$.

Multivariate analyses. In order to identify those variables associated with variation in Gall serum levels we fitted a multivariate analysis using generalised linear models nested by patient and visits using the xtgee command of STATA. The population-averaged generalised estimating equations were first modelled by adding all variables with a $p$ value $<0.15$ to the bivariate analysis. The final models were constructed using quasi-likelihood estimation based on the independence model information criterion ${ }^{32}$ and Wald tests, removing all variables with $p>0.15$. The variable time of frozen storage was always included in the multivariate analysis. Once the best model was obtained, several variables related with assessment of RA were forced in the model in order to determine whether there was an association with Gall or not.

In addition, to determine whether there was an association between Gall and IL6 serum levels, we fitted another multivariate analysis using xtgee command of Stata as described above, but in this case the dependent variable was IL6 serum levels, which did not follow a normal distribution, so the variable was transformed through double square root transformation. The best model was obtained as described above.

In order to assess the ability of Gall serum levels to discriminate between RA patients and healthy donors or between RA and UA patients we generated Receiver Operating Characteristic (ROC) curves through the command roctab and the option graph. Each cut-off point was selected on the basis of the best trade-off values between sensitivity, specificity, cases correctly classified and positive (LR+) and negative (LR-) likelihood ratios reported using the command roctab with the option detail.

Ethical considerations. PEARL study is conducted according to the principles expressed in the Helsinki Declaration of 1983 and it was approved by the Research Ethics Committee of Hospital Universitario de La Princesa (PI-518; March 28th, 2011). All patients included were over 18 years old and signed a written informed consent at study entry and samples and data from patients included in this study were provided by the IISIP Biobank (ISCIII B.0000763). All samples were processed following standard operating procedures with the appropriate approval of the Ethics and Scientific Committees.

\section{Data availability}

All raw data from serum and synovial fluid required to reproduce our results are provided as the Supplementary files "serum.xlsx" and "synovial.xlsx". Any issue regarding the interpretation of the variables, please contact with Dr. Isidoro González-Âlvaro: isidoro.ga@ser.es

Received: 15 June 2020; Accepted: 21 September 2020

Published online: 20 October 2020

\section{References}

1. McInnes, I. B. \& Schett, G. Pathogenetic insights from the treatment of rheumatoid arthritis. Lancet 389, 2328-2337 (2017). 
2. Schoels, M. et al. Evidence for treating rheumatoid arthritis to target: Results of a systematic literature search. Ann. Rheum. Dis. 69, 638-643 (2010).

3. Vaishnaw, A. K., McNally, J. D. \& Elkon, K. B. Apoptosis in the rheumatic diseases. Arthritis Rheum. 40, 1917-1927 (1997).

4. Firestein, G. S. \& McInnes, I. B. Immunopathogenesis of rheumatoid arthritis. Immunity 46, 183-196 (2017).

5. Okada, Y. et al. Genetics of rheumatoid arthritis contributes to biology and drug discovery. Nature 506, 376-381 (2014).

6. Smolen, J. S. \& Aletaha, D. Forget personalised medicine and focus on abating disease activity. Ann. Rheum. Dis. 72, 3-6 (2013).

7. Landewe, R. B. M. Overdiagnosis and overtreatment in rheumatology: A little caution is in order. Ann. Rheum. Dis. 77, 1394-1396 (2018).

8. Seoane, I. V. et al. Vasoactive intestinal peptide gene polymorphisms, associated with its serum levels, predict treatment requirements in early rheumatoid arthritis. Sci. Rep. 8, 2035 (2018).

9. Gonzalez-Alvaro, I. et al. Biomarkers predicting a need for intensive treatment in patients with early arthritis. Curr. Pharm. Des. 21, 170-181 (2015).

10. Barondes, S. H., Cooper, D. N., Gitt, M. A. \& Leffler, H. Galectins. Structure and function of a large family of animal lectins. J. Biol. Chem. 269, 20807-20820810 (1994).

11. Hirabayashi, J. \& Kasai, K. The family of metazoan metal-independent beta-galactoside-binding lectins: Structure, function and molecular evolution. Glycobiology 3, 297-304 (1993).

12. Rubinstein, N., Ilarregui, J. M., Toscano, M. A. \& Rabinovich, G. A. The role of galectins in the initiation, amplification and resolution of the inflammatory response. Tissue Antigens 64, 1-12 (2004).

13. Perillo, N. L., Pace, K. E., Seilhamer, J. J. \& Baum, L. G. Apoptosis of T cells mediated by galectin-1. Nature 378, 736-739 (1995).

14. Toscano, M. A. et al. Differential glycosylation of TH1, TH2 and TH-17 effector cells selectively regulates susceptibility to cell death. Nat. Immunol. 8, 825-834 (2007).

15. Rabinovich, G. A. et al. Recombinant galectin-1 and its genetic delivery suppress collagen-induced arthritis via $\mathrm{T}$ cell apoptosis. J. Exp. Med. 190, 385-398 (1999).

16. Chung, C. D., Patel, V. P., Moran, M., Lewis, L. A. \& Miceli, M. C. Galectin-1 induces partial TCR zeta-chain phosphorylation and antagonizes processive TCR signal transduction. J. Immunol. 165, 3722-3729 (2000).

17. Rabinovich, G. A. et al. Specific inhibition of T-cell adhesion to extracellular matrix and proinflammatory cytokine secretion by human recombinant galectin-1. Immunology 97, 100-106 (1999).

18. Mendez-Huergo, S. P. et al. Clinical relevance of galectin-1 and galectin-3 in rheumatoid arthritis patients: Differential regulation and correlation with disease activity. Front. Immunol. 9, 3057 (2018).

19. Xibille-Friedmann, D., Bustos Rivera-Bahena, C., Rojas-Serrano, J., Burgos-Vargas, R. \& Montiel-Hernandez, J. L. A decrease in galectin-1 (Gal-1) levels correlates with an increase in anti-Gal-1 antibodies at the synovial level in patients with rheumatoid arthritis. Scand. J. Rheumatol. 42, 102-107 (2013).

20. Harjacek, M. et al. Expression of galectins- 1 and -3 correlates with defective mononuclear cell apoptosis in patients with juvenile idiopathic arthritis. J. Rheumatol. 28, 1914-1922 (2001).

21. Dasuri, K. et al. The synovial proteome: Analysis of fibroblast-like synoviocytes. Arthritis Res. Ther. 6, R161-R168 (2004).

22. Gomez-Chavez, F. et al. Galectin-1 reduced the effect of LPS on the IL-6 production in decidual cells by inhibiting LPS on the stimulation of IkappaBzeta. J. Reprod. Immunol. 112, 46-52 (2015).

23. Municio, C. et al. Methotrexate limits inflammation through an A20-dependent cross-tolerance mechanism. Ann. Rheum. Dis. 77, 752-759 (2018).

24. Lamana, A. et al. The TT genotype of the STAT4 rs7574865 polymorphism is associated with high disease activity and disability in patients with early arthritis. PLoS ONE 7, e43661 (2012).

25. Smolen, J. S. et al. EULAR recommendations for the management of rheumatoid arthritis with synthetic and biological diseasemodifying antirheumatic drugs: 2019 update. Ann. Rheum. Dis. 79, 685-699 (2020).

26. Aletaha, D. et al. 2010 rheumatoid arthritis classification criteria: An American College of Rheumatology/European League against rheumatism collaborative initiative. Ann. Rheum. Dis. 69, 1580-1588 (2010).

27. Regueiro, C. et al. Value of measuring anti-carbamylated protein antibodies for classification on early arthritis patients. Sci. Rep. 7, 12023 (2017).

28. Gonzalez-Alvaro, I. et al. Interleukin 15 levels in serum may predict a severe disease course in patients with early arthritis. PLoS ONE 6, e29492 (2011).

29. Arnett, F. C. et al. The American Rheumatism Association 1987 revised criteria for the classification of rheumatoid arthritis. Arthritis Rheum. 31, 315-324 (1988).

30. Verpoort, K. N. et al. Undifferentiated arthritis-disease course assessed in several inception cohorts. Clin. Exp. Rheumatol. 22, S12-S17 (2004).

31. Team, R. C. A language and environment for statistical computing. (R Foundation for Statistical Computing, Vienna, 2020) https ://www.R-project.org/.

32. Pan, W. Akaike's information criterion in generalized estimating equations. Biometrics 57, 120-125 (2001).

\section{Acknowledgements}

We thank the patients and the controls for their participation in the study. Also we are grateful to collaborating clinicians for their participation in this study. We also acknowledge the excellent editing assistance of Dr. Manuel Gomez Gutierrez.

This work was supported by grants RD16/0011/0012 and PI18/0371 to IG-A, and PI12/01578 to SC from the Ministerio de Economía y Competitividad (Instituto de Salud Carlos III) and co-funded by Fondo Europeo de Desarrollo Regional (FEDER). A.T-M is currently granted by a PhD fellowship from the Autonomous Region of Madrid (PEJD-2019-PRE/BMD-16851). NM is currently granted by a RD16/0011/0012. The work of ER-V is currently by a grant Rio-Hortega CM19/00149 from the Ministerio de Economía y Competitividad (Instituto de Salud Carlos III) and co-funded by Fondo Europeo de Desarrollo Regional (FEDER).

\section{Author contributions}

A.T.-M., H.d.l.F., A.L and I.G.-A. designed the study. A.T.-M., E.R.-V. and A.L. performed laboratory determinations. A.M.O., S.C. and I.G.-A. provide patients and clinical and laboratory data. A.T.-M., N.M., A.L. and I.G.-A. performed analysis of the results, interpreted the results and wrote the first draft of the manuscript. H.d.l.F., E.R.-V., A.M.O. and S.C. revised the manuscript. All authors approved the final version of the manuscript.

\section{Competing interests}

IG-A reports grants from Instituto de Salud Carlos III, during the course of the study; personal fees from Lilly and Sanofi; personal fees and non-financial support from BMS; personal fees and non-financial support from 
Abbvie; research support, personal fees and non-financial support from Roche Laboratories; non-financial support from MSD, Pfizer and Novartis, not related to the submitted work.

SC has received personal fees from Amgen, Lilly, MSD, Pfizer, Roche, Sanofi and SOBI. SC is also assistant professor of the cátedra UAM-Roche, EPID-Future, Universidad Autónoma de Madrid (UAM).

The remaining authors report no competing interests with the submitted work.

\section{Additional information}

Supplementary information is available for this paper at https://doi.org/10.1038/s41598-020-74185-8.

Correspondence and requests for materials should be addressed to I.G.-A. or A.L.

Reprints and permissions information is available at www.nature.com/reprints.

Publisher's note Springer Nature remains neutral with regard to jurisdictional claims in published maps and institutional affiliations.

(c) (i) Open Access This article is licensed under a Creative Commons Attribution 4.0 International License, which permits use, sharing, adaptation, distribution and reproduction in any medium or format, as long as you give appropriate credit to the original author(s) and the source, provide a link to the Creative Commons licence, and indicate if changes were made. The images or other third party material in this article are included in the article's Creative Commons licence, unless indicated otherwise in a credit line to the material. If material is not included in the article's Creative Commons licence and your intended use is not permitted by statutory regulation or exceeds the permitted use, you will need to obtain permission directly from the copyright holder. To view a copy of this licence, visit http://creativecommons.org/licenses/by/4.0/.

(C) The Author(s) 2020 